\title{
Gestion de la qualité dans \\ la transformation laitière : expérimentation d'une démarche d'élaboration concertée de guides de bonnes pratiques d'hygiène au Sénégal et au Burkina Faso
}

\author{
C. Broutin ${ }^{1 *}$ M. François ${ }^{2}$ N. La Noë Niculescu ${ }^{2}$
}

\begin{abstract}
Mots-clés
Lait - Produit laitier - Qualité -

Hygiène - Sénégal - Burkina Faso.
\end{abstract}

\begin{abstract}
Résumé
La transformation du lait à petite échelle se développe en Afrique de l'Ouest. Elle permet de valoriser la production laitière locale et de répondre aux besoins et attentes des acteurs. Le succès de ces nouvelles petites entreprises est cependant tributaire d'une meilleure maîtrise de la qualité dans ces entreprises. Une démarche d'élaboration de guides de bonnes pratiques d'hygiène pour la transformation laitière, basée sur la concertation entre les professionnels, les services de l'Etat et avec la collaboration des autres acteurs institutionnels (laboratoires, recherche, développement, normalisation, consommateurs), tous regroupés au sein d'un comité de suivi, a été mise en œuvre au Sénégal et au Burkina Faso. L'élaboration des guides a reposé sur une série d'étapes validées par le comité de suivi assurant le cadrage général de l'étude. Les échanges ont permis d'aborder la faiblesse des données sur les risques sanitaires, l'évolution nécessaire de la réglementation, des normes et des pratiques mises en œuvre par les acteurs de la filière. Dans ce processus d'élaboration d'un certain nombre de procédures et de pratiques adaptées et applicables par les acteurs, les points de vue des experts comme celui des acteurs de terrain ont été pris en compte. Pour que de tels guides contribuent effectivement à créer un environnement propice au développement de la filière, ils devront être largement diffusés et transposés en supports d'information et de formation pour tous les acteurs de la filière lait, les organismes d'appui et les agents de I'Etat. II est également nécessaire de réfléchir aux modalités qui permettront que son application puisse donner aux professionnels une reconnaissance de qualité, comme la création de marque commerciale collective ou de labels.
\end{abstract}

1. Gret - antenne Dakar, Dakar, Sénégal.

2. Gret, Nogent-sur-Marne, France.

* Auteur pour la correspondance

Gret, Campus du Jardin Tropical, 45 bis avenue de la Belle Gabrielle, 94736 Nogent-sur-Marne Cedex, France.

E-mail : broutin@gret.org
INTRODUCTION : NECESSITE POUR LES ACTEURS DES FILIERES LOCALES DE MAITRISER LA QUALITE SANITAIRE DE LEURS PRODUITS

Après les nombreux échecs des laiteries industrielles, la transformation du lait à petite échelle se développe en Afrique de l'Ouest pour valoriser la production locale et répondre aux besoins et attentes des consommateurs (6). Le développement de ce secteur constitue un enjeu important en raison notamment des revenus induits par cette activité tant pour les acteurs de la transformation que pour les éleveurs. Il est cependant tributaire d'une meilleure maitrise technique de la production et de la transformation. En particulier, l'essor de ces filières artisanales passe par l'amélioration de la qualité dans ces unités laitières ainsi qu'en amont (au niveau 
de la gestion du cheptel, de la traite et de la collecte) et en aval (au niveau du circuit de distribution et de la consommation) (1).

Le lait est en effet un produit fragile, susceptible d'être altéré par de nombreuses réactions chimiques et microbiologiques. Le principal danger, qui doit entraîner une grande vigilance des professionnels, est l'apparition de toxi-infections alimentaires collectives dues à l'ingestion de produits laitiers impropres à la consommation. Elles sont liées à la contamination de ces produits par un agent infectieux, la multiplication et/ou la survie de micro-organismes dans des conditions favorables. Le cheptel, l'environnement, le matériel, les conditionnements, les matières premières et le personnel représentent tous une source potentielle de contamination. Les diverses pratiques et procédés de production, de transformation et de conservation du lait constituent autant de paramètres à prendre en compte pour limiter le développement de ces agents infectieux (1).

Les efforts collectifs d'amélioration de la qualité peuvent être analysés comme le résultat d'arbitrages entre les exigences de santé publique et les règles de la concurrence (14). Pour des produits à forts enjeux sanitaires comme le lait, la démarche des pouvoirs publics est en général de privilégier des politiques de qualité très coercitives, s'appuyant sur des réglementations contraignantes destinées à protéger le consommateur. Cependant, en Afrique, la mise en place de ce type de politique butte sur la faiblesse des services de contrôle et de répression $(1,6)$. Dans ce contexte, plusieurs projets de développement s'appuient de manière croissante sur des démarches d'incitation à l'autocontrôle de la qualité par les entreprises ou les organisations professionnelles elles-mêmes.

La mise en place d'une politique de qualité dans les entreprises, même les plus petites, est donc une priorité en termes de protection du consommateur. Les réglementations en vigueur consacrent d'ailleurs de plus en plus la responsabilité active du fabricant qui doit mettre en place les moyens lui permettant d'assurer la sécurité et la salubrité des denrées alimentaires qu'il met sur le marché $(11,15)$.

Le Codex Alimentarius (15) définit dans ses principes généraux d'hygiène alimentaire les principes à adopter pour les industries de transformation alimentaire. Ces textes sont basés sur l'utilisation de la méthode d'analyse de danger des points critiques (hazard analysis of critical control points ; Haccp), mais ils prévoient également le recours aux guides de bonnes pratiques (good manufacturing practices) pour définir dans chaque secteur de production, selon sa spécificité, les bonnes pratiques de fabrication permettant d'assurer la sécurité du consommateur. Les guides de bonnes pratiques d'hygiène sont axés sur la sécurité des aliments. Ils permettent à certains industriels de disposer d'éléments pratiques pour initier une démarche Haccp détaillée et adaptée à leur entreprise. Pour l'artisanat et la transformation à petite échelle, ils fournissent des éléments de mâ̂trise concrets adaptés à leur structure d'entreprise (11).

Cette approche de guides de bonnes pratiques par secteur d'activité peut être mobilisée par les petites entreprises de transformation agroalimentaire pour que la spécificité de leurs conditions de production, des risques correspondant à leur activité, soit mieux prise en compte à terme dans les réglementations nationales et internationales *. Actuellement, ces petites entreprises contournent souvent la réglementation sans pour autant mettre en danger le consommateur ; mais n'ayant pas été impliquées dans le processus d'élaboration des lois, ces entreprises considèrent que les régle-

* Par exemple, les fabricants de fromages fermiers en France ont fait reconnaitre un guide de bonnes pratiques en transformation fermière. Ce guide prend en compte une analyse des risques et moyens de leur maîtrise (démarche Haccp) spécifiques des petites unités de transformation. mentations en vigueur sont très souvent obsolètes ou inapplicables dans leur domaine d'activité.

Dans beaucoup de pays en développement (7), la transformation des produits alimentaires est tributaire d'un grand nombre de petits producteurs. Bien que cette organisation puisse comporter des avantages socio-économiques, elle conduit aussi à des niveaux de contamination élevés du fait du caractère souvent très rudimentaire des installations ou du manque de formation du personnel en hygiène alimentaire. Comme le souligne l'Organisation mondiale de la santé, cela ne veut pas dire que tous les aliments de ce type sont insalubres : nombre de pratiques traditionnelles de production ou de consommation des aliments comportent des marges de sécurité intrinsèques, fondées sur des années d'expérience (15). Par exemple, certaines petites unités de transformation laitière traitent le lait rapidement après la collecte, ou utilisent des techniques ou des procédés favorables à la sécurité alimentaire (par exemple la fermentation rapide du lait conférant un niveau d'acidité élevé, défavorable au développement de bactéries pathogènes). Cependant, de nouveaux types de problèmes peuvent survenir suite à l'introduction de nouvelles technologies (qui accroissent les possibilités techniques des transformateurs) et à l'urbanisation de plus en plus poussée. En effet, lorsque la distance entre le producteur et le consommateur augmente, il est plus difficile au consommateur d'évaluer la qualité des aliments achetés, surtout lorsque le système de contrôle et d'étiquetage des produits alimentaires est très peu développé, comme c'est le cas dans une grande partie des pays africains. Dans ce contexte, des procédures d'autocontrôle de la qualité par les unités elles-mêmes sont susceptibles d'améliorer la confiance entre partenaires des transactions et de diminuer les risques sanitaires liés à la consommation des produits.

Ainsi, les guides de bonnes pratiques constituent un des outils à la disposition des acteurs économiques, petites entreprises de transformation agroalimentaires, pour améliorer collectivement la qualité des produits mis sur le marché et faire reconnaître au niveau de l'Etat la validité de leur démarche.

L'élaboration de guides de bonnes pratiques d'hygiène repose sur la démarche Haccp, qui inclut une analyse des dangers, la détermination des points clés et des pratiques pour leur maîtrise, et enfin la définition des éléments (ou paramètres) de surveillance. L'élaboration de tels guides permet de définir, de manière concertée, avec les professionnels d'un secteur, les services de l'Etat et la collaboration des autres acteurs institutionnels (laboratoires, recherche, développement, normalisation, association de consommateurs) les pratiques adaptées au contexte pour garantir la sécurité sanitaire des consommateurs.

Cette démarche a été mise en œuvre au Sénégal et au Burkina Faso avec l'appui du Gret, qui dispose d'une expérience dans ce domaine pour les produits fermiers en Europe *, et de projets de la coopération française : le Projet d'appui au développement durable et de lutte contre la pauvreté en milieu rural (Pamir), le Projet d'appui au renforcement institutionnel des organisations professionnelles d'éleveurs modernes (Ariope), le Projet d'appui à l'environnement institutionnel et au développement du secteur privé (EIDév) au Burkina Faso, et le Fonds de soutien prioritaire (FSP) «Actions régionales pour la qualité dans le secteur agroalimentaire en Afrique ». L'objectif de cette communication n'est pas de présenter en détail les guides ** mais de mettre l'accent sur quelques constats et résultats de la démarche qui a été adoptée, et de présenter en

\footnotetext{
* Guide de bonnes pratiques transformation fermière et Guide de bonnes pratiques d'hygiène pour la transformation et la dégustation en fermes pédagogiques (à paraitre au Journal officiel, France).

** http://www.gret.org/ressource/result_nouveau.asp
} 
conclusion quelques perspectives dans le cadre de la réflexion sur les politiques laitières.

\section{MATERIEL ET METHODES :}

EXPERIENCE DES GUIDES DE BONNES PRATIQUES AU SENEGAL ET AU BURKINA FASO

La méthode d'élaboration des guides de bonnes pratiques d'hygiène laitière au Sénégal et au Burkina Faso est schématisée dans la figure 1.

La réalisation d'un tel guide dans chaque pays a dû associer quatre types de partenaires, au sein d'un comité de suivi, mis en place dans les deux pays au démarrage des travaux en janvier 2005 : les professionnels de la filière lait (éleveurs, collecteurs et transformateurs), et leurs organisations professionnelles les plus importantes et représentatives; les services de l'Etat (services vétérinaires et services de l'élevage, services d'hygiène et de contrôle des denrées alimentaires, services de normalisation) ; des représentants d'organismes d'appui, de recherche, des personnes ressources qui ont pu fournir un avis aux différentes étapes de l'élaboration du guide ; enfin, une expertise dans le domaine de la transformation et de la qualité des produits laitiers qui a animé et alimenté la concertation - cette expertise a été fournie par le Gret.

Le comité de suivi a eu pour rôle d'assurer un cadrage général de l'étude et de valider la méthodologie proposée, d'examiner et de valider les résultats des différentes étapes de l'étude, d'appuyer l'organisation des ateliers de restitution (national et sous-régional), et de mettre en œuvre les recommandations finales, notamment la valorisation et la diffusion du guide, et la recherche des partenaires pour son application. L'élaboration des guides a reposé sur l'enchaînement des étapes suivantes :

- la détermination du champ d'application (produits, entreprises) ;

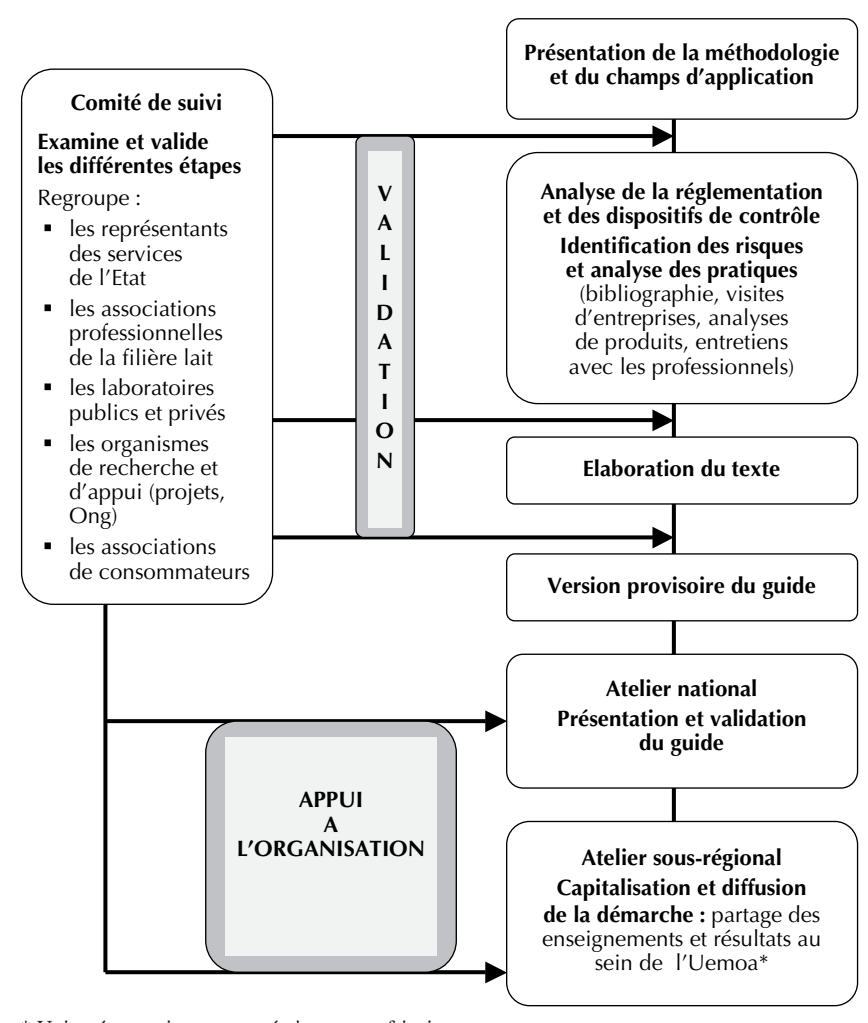

* Union économique et monétaire ouest-africaine

Figure 1 : démarche d'élaboration des guides.
- une analyse bibliographique pour recueillir toutes les données, études et résultats de recherche disponibles ;

- une analyse de la réglementation et des dispositifs de contrôle ;

- une identification des risques et la définition de règles de bonnes pratiques « accessibles » aux acteurs de la filière, à partir des ressources documentaires, de visites de terrain (entreprises, collecteurs, éleveurs), d'entretiens avec les professionnels et, si nécessaire, d'analyses microbiologiques, qui sont validées par le comité de suivi :

- l'élaboration du texte par l'expertise technique mobilisée (équipe du Gret), texte qui a été validé par le comité de suivi et ensuite par un atelier national en novembre $2005(4,5)$.

Pour favoriser les échanges entre acteurs de différents pays de l'Union économique et monétaire ouest-africaine (Uemoa) sur la démarche, un atelier sous-régional a été organisé à Ouagadougou en décembre 2005 (10) où le Bénin, le Burkina Faso, le Mali, le Niger et le Sénégal étaient représentés.

\section{RESULTATS ET DISCUSSION : DIMENSIONS TECHNIQUES ET ORGANISATIONNELLES DU GUIDE DE BONNE PRATIQUES}

\section{Nécessité de disposer de données précises sur la qualité sanitaire du lait et des produits laitiers}

Dans les deux pays, les travaux menés par l'administration, les projets, les organismes de recherche et de développement ont permis de disposer de données sur la filière lait et plus spécifiquement sur le secteur de la transformation. Ils ont mis en évidence le développement de ce secteur au cours des dernières années. Ainsi au Sénégal, les informations sur le nombre de petites entreprises, leur localisation, leurs activités $(2,3,9)$ ont montré l'essor des minilaiteries (une dizaine en 2000 et une quarantaine en 2005) mais également de la transformation artisanale et industrielle du lait en poudre. Au Burkina Faso, la transformation des produits laitiers est également apparue comme une activité en pleine expansion. En 2005, on a dénombré 73 unités de transformation dans la seule ville d'Ouagadougou et 12 à Bobo Dioulasso (13). Ces unités utilisent du lait cru provenant des petites fermes périurbaines ou du lait en poudre d'importation. En zone urbaine, la transformation du lait en yaourt et en lait caillé est une source d'emploi et de revenus pour de nombreuses familles.

Les recherches bibliographiques ont cependant mis en exergue le manque de données et de travaux de recherche sur la qualité du lait (notamment sur l'importance des différentes zoonoses) et des produits laitiers. Ce constat a amené le comité de suivi au Sénégal à proposer à la coopération française la réalisation d'une étude sur la qualité des produits laitiers cordonnée par l'Institut Pasteur (Breurec et coll., article soumis à revue anglophone). Quelques analyses microbiologiques ont également été réalisées au Burkina et EIDév a financé une étude sur la date limite de consommation pour le yaourt, réalisée par le Laboratoire national de santé publique. Des travaux d'études des barèmes de pasteurisation, de la date limite de consommation du lait caillé et de la composition chimique des produits laitiers au Sénégal ont été réalisés par le Laboratoire d'analyses et d'essais de l'Université de Dakar et utilisés lors de l'élaboration du guide.

Dans les autres pays qui ont participé à l'atelier sous-régional, le même constat a été fait : trop peu de données sur la qualité du lait et des produits laitiers sont disponibles. Dans l'avenir, des analyses devront être conduites pour appuyer la mise en place de démarches qualité. 


\section{Réglementation souvent obsolète ou peu adaptée, performance des systèmes de contrôle à améliorer}

Dans certains pays, comme au Sénégal, la complexité du système de contrôle des denrées alimentaires a été soulignée. Ce système mobilise en effet différents services de plusieurs ministères sans que les rôles et prérogatives de chacun soient clairs, reconnus et connus des autres. La réglementation, quand elle existe, est souvent ancienne, copiée sur celle de pays européens et est donc en partie ni applicable, ni appliquée car elle ne correspond pas à l'environnement de production et de transformation de ces pays. Le principal texte au Sénégal date de 1969 (décret 69-891 du 25 juillet 1969, réglementant le contrôle du lait et des produits exemple, que la pasteurisation doit être pratiquée « dans un appareil ou un groupe d'appareils appropriés et correctement utilisés selon un principe approuvé par la direction de l'Elevage après avis de la commission de contrôle des produits alimentaires ». Or aucun texte n'a été trouvé concernant les principes approuvés, hormis ceux mentionnés dans le décret (chauffage à $63{ }^{\circ} \mathrm{C}$ pendant 30 min et chauffage instantané à $95^{\circ} \mathrm{C}$ ). Il est également indiqué que les appareils doivent être munis d'enregistreurs de températures ( "graphiques datés, classés et conservés pendant six mois »), alors que le chauffage, dans les minilaiteries, est effectué au bain-marie dans des marmites et que la pasteurisation à basse température $\left(63{ }^{\circ} \mathrm{C}\right)$ n'a pas donné de résultats satisfaisants lors des tests réalisés par le Laboratoire d'analyses et d'essais. Le décret 2002 - 1094 du 4 novembre 2002 stipule que, pour la plupart des maladies, « l'autorité administrative compétente, sur proposition du service de l'élevage, prend un arrêté portant déclaration d'infection ». Or, ces services n'ont eu ni les moyens de faire un suivi et un contrôle des troupeaux, ni de prendre ces arrêtés et d'en vérifier l'application. Les éleveurs n'ont pas eu non plus les moyens d'appliquer la réglementation (isolement des animaux, traitement aux frais de l'éleveur et parfois abattage des animaux) et aucune indemnisation n'est prévue pour les inciter à le faire.

Six normes ont été identifiées pour les produits laitiers au Sénégal, mais elles sont souvent anciennes (1980-90), parfois obsolètes ou incomplètes (absence de critères microbiologiques pour le lait cru), très difficiles à atteindre. Au Burkina Faso, il n'existait aucune norme de ce type en 2005. Si les normes internationales constituent des objectifs de qualité à atteindre, elles nécessitent une adaptation progressive des systèmes de production et de transformation (évolution par «paliers » comme cela a été le cas en Europe) et doivent être complétées pour les produits laitiers traditionnels. Il s'avère donc nécessaire, en utilisant le cadre de concertation constitué par le comité de suivi, d'envisager une évolution de la réglementation (révision, textes complémentaires adaptés aux différents niveaux de transformation) et un renforcement des compétences et des moyens des services publics et organismes privés.

\section{Dangers surtout microbiologiques et moyens de maîtrise à la portée des minilaiteries}

Comme signalé en introduction, le principal danger est d'ordre microbiologique (contamination par un agent infectieux et multiplication et/ou survie de micro-organismes dans des conditions favorables). Cet agent infectieux peut être apporté par le cheptel, l'environnement, le matériel, les conditionnements, les matières premières et le personnel. Les pratiques et procédés de transformation et de conservation des aliments pourront ensuite créer des conditions favorables à leur développement. Les autres principaux dangers (chimiques et physiques) proviennent : soit de la présence éventuelle de résidus de produits de traitements zoosanitaires, de produits chimiques dangereux dans les matières premières, de produits chimiques dangereux dans l'atelier ; soit de la présence d'impuretés dans le lait ou les matières premières ; soit de la sensibilité particulière aux infections de certains (personnes immunodéprimées, femmes enceintes, bébés, personnes très âgées, personnes non vaccinées...).

Les guides ont été élaborés pour les micro- et petites entreprises artisanales et semi-industrielles transformant le lait cru ou le lait en poudre en lait pasteurisé, lait caillé, yaourt pour les aider à maîtriser ces risques. Leur élaboration a reposé sur deux piliers essentiels :

- la maitrise des dangers liés à la transformation du lait par la compétence des opérateurs. Le transformateur doit être parfaitement au courant des risques alimentaires et de la façon de les prévenir. Une approche par la formation du personnel responsable des unités de transformation a été privilégiée (par rapport à une démarche d'investissement dans des locaux et/ou dans des équipements particuliers) ;

- la maîtrise de la qualité du lait, matière première, par la pasteurisation (lait local et lait reconstitué à partir de poudre de lait). Pour assurer la sécurité du consommateur, il est préconisé que l'unité de transformation pasteurise systématiquement le lait avant la transformation. Cette recommandation reste un facteur de sécurité important pour le consommateur, particulièrement en cas d'utilisation du lait cru. La pasteurisation ne saurait cependant dispenser le transformateur d'une attention soutenue portée à la qualité du lait acheté aux éleveurs. En particulier, le transformateur doit chercher, autant que possible, à sensibiliser les éleveurs avec lesquels il travaille sur l'importance du suivi de la santé du cheptel (surveillance de la brucellose, tuberculose, mammites en particulier) et des conditions de traite. Mais, si une défaillance apparaît à ce niveau, la pasteurisation permet, si elle est bien conduite, en respectant scrupuleusement le couple temps-température de pasteurisation, d'assurer la sécurité du consommateur.

En conséquence, les recommandations ont privilégié des gestes d'hygiène simples (travail dans un environnement et avec du matériel propre et désinfecté, lavage des mains avant la transformation, vêtements adaptés, évacuation des déchets, maintien de la chaîne du froid...) et l'utilisation d'équipements courants maintenus dans un parfait état de propreté $(8,12)$. Les guides se composent : d'une analyse synthétique des dangers basée sur l'expérience des professionnels et le concours de spécialistes extérieurs, de « points clés » et d' "éléments de surveillance » (illustrés), enfin de « fiches de bonnes pratiques » générales, par opération unitaire et par produit, visant la maîtrise des dangers.

\section{Expérience innovante de concertation et de négociation entre les acteurs}

La démarche d'élaboration des guides de bonnes pratiques d'hygiène a constitué une expérience innovante de concertation et de négociation entre les acteurs de la filière, les organismes d'appui et de recherche, et l'Etat. En effet, pour aborder le problème des risques sanitaires, il a fallu mettre en perspective les réglementations, les normes et les pratiques mises en œuvre dans la réalité par les acteurs de la filière, pour élaborer un certain nombre de procédures et de pratiques applicables par les acteurs en tenant compte de la réalité locale et permettant d'accéder à des niveaux de qualité satisfaisants. Dans ce processus, le point de vue des acteurs de terrain a été aussi important que celui des experts. Les discussions lors des réunions des comités de suivi et lors des ateliers nationaux de validation l'ont montré. Le produit obtenu (guide) est aussi important que le processus et la concertation qui ont permis son élaboration. Ce travail a nécessité du temps (presque une année) et a permis également aux agents de l'Etat de prendre conscience du 
développement de ces minilaiteries, pas toujours très visible et méconnu des fonctionnaires travaillant dans les administrations centrales des capitales.

\section{Occasion de rencontre entre acteurs de la sous-région}

L'atelier sous-régional organisé en décembre 2005 a été l'occasion pour les acteurs des différents pays de se rencontrer, et d'échanger sur la situation de la filière, de la réglementation, de la qualité des produits dans les différents pays et sur l'intérêt de telles démarches de concertation et de supports de maitrise de la qualité pour des petites entreprises. Il leur a également permis de prendre conscience de l'importance de l'Uemoa au niveau duquel les directives réglementaires et les priorités politiques sont définies.

\section{Plus de 800 guides diffusés et près de 10000 téléchargements du fichier}

Suite à l'atelier sous-régional de présentation de la démarche d'élaboration des guides aux acteurs de la sous-région, plus de 400 guides ont été diffusés dans chaque pays (figure 2). Outre les participants aux ateliers, les laiteries référencées dans le répertoire des entreprises agroalimentaires de la Chambre de commerce d'industrie et d'artisanat d'Ouagadougou et les membres des organisations

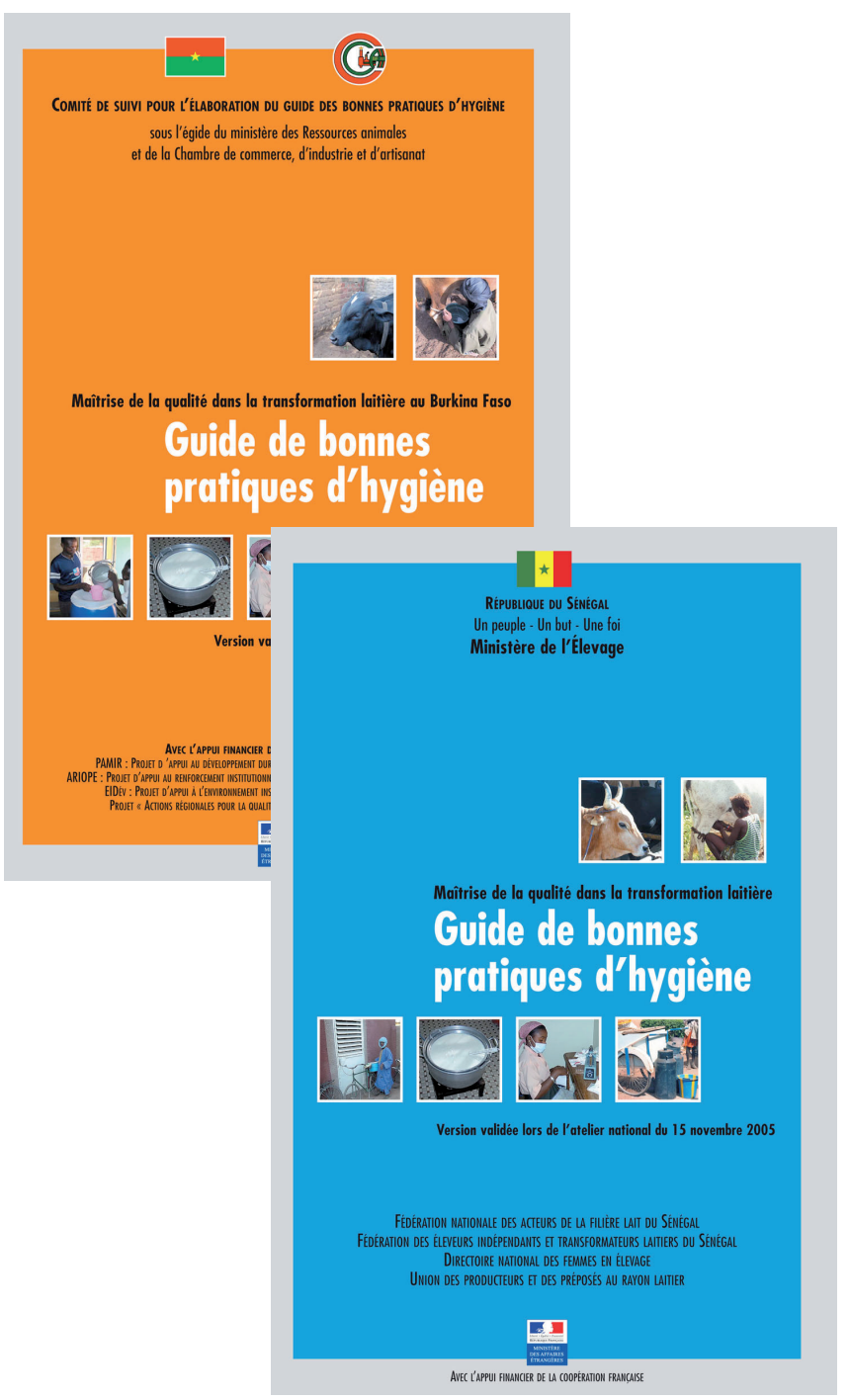

Figure 2 : pages de couverture des deux guides. professionnelles au Sénégal ont reçu un exemplaire du guide. Les projets ayant apporté un soutien financier ont assuré leur propre diffusion, généralement au sein des services de l'Etat et des opérateurs. Le guide a également été présenté à diverses occasions comme au Siagro à Dakar en avril 2006, à l'atelier du Réseau sur les politiques laitières à Bamako en juillet 2006, à l'atelier de concertation de la filière lors de la « journée du lait » au Sénégal en juin 2007. Des affiches illustrant les bonnes pratiques et différentes analyses ont été réalisées et diffusées dans les laiteries au Sénégal et au Burkina (figure 3). Ces affiches reprennent les étapes essentielles identifiées dans le guide comme points critiques.

Pour assurer une plus large diffusion, les guides ont été mis en téléchargement sur les sites du Gret, d'Infoconseil au Sénégal et du réseau sur les politiques laitières *. En mars 2008, le guide du Sénégal a été téléchargé près de 7400 fois et celui du Burkina près de 2000 fois sur le site du Gret. Les guides n'ont toutefois pas fait l'objet de cession de formation ni de réel suivi de leur application. Les auteurs ne sont donc pas en mesure de préciser l'impact sur la qualité finale des produits transformés. Au Sénégal, les autorités et les associations de producteurs ont décidé de s'engager dans une démarche qualité avec la mise en place envisagée d'un label soustendu par l'application du Guide de bonnes pratiques. Un projet mis en œuvre par le Gret, le Groupe de recherche action formation (Enda Graf, ONG sénégalaise) et la Fédération nationale des acteurs de la filière lait local du Sénégal (Fenafils) a démarré courant 2008 pour encourager cette démarche avec l'appui financier de la coopération française et de l'Etat sénégalais.

\footnotetext{
* www.gret.org, www.infoconseil.sn, www.repol.info
}

\section{Qualité des produits laitiers : bonnes pratiques d'hygiène Livraison rapide et filtration du lait}
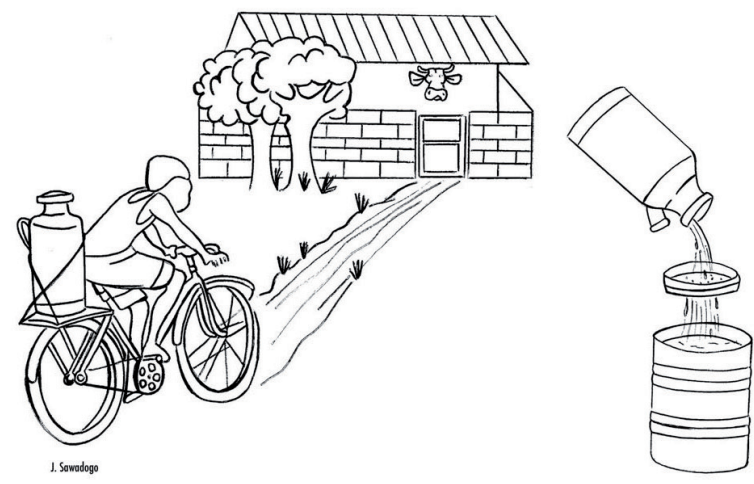

- Acheminer rapidement le lait après la traite (4 h maximum entre la traite et la pasteurisation si le transport n'est pas réfrigéré) dans des bidons à large ouverture.

- À la laiterie, filtrer le lait pour éliminer les impuretés avec un filtre à usage unique, ou avec un linge lavé et désinfecté après chaque filtration.

- Effectuer les tests de contrôle de la qualité

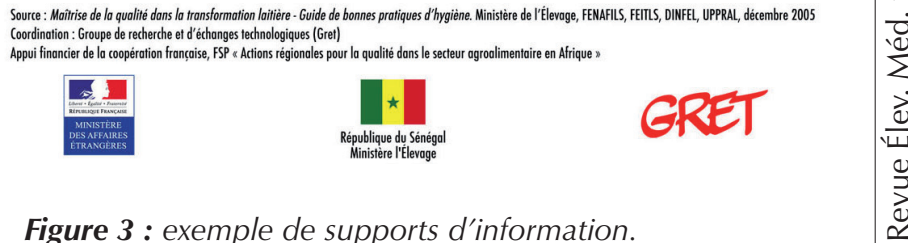


CONCLUSION :

AU-DELA DU GUIDE, EXISTENCE D'UN CADRE DE CONCERTATION SUR LA QUALITE

\section{Guide de bonnes pratiques d'hygiène : support qui doit être valorisé par la production de supports, des formations et du conseil}

Validés par les pouvoirs publics, des professionnels, des chercheurs, des agents de développement, des consommateurs, des responsables de laboratoires et de l'institut de normalisation et de l'Etat, ces guides demeurent cependant d'application volontaire. Pour qu'ils contribuent effectivement à créer un environnement propice au développement de la transformation du lait local, ils devront tout d'abord être largement diffusés et transposés en supports d'information (fiches didactiques, affichettes, produits audiovisuels, notamment en langues nationales) pour tous les acteurs de la filière lait, les organismes d'appui et les agents de l'Etat. Il est également nécessaire de concevoir et de tester des modules de formation pour les différents types d'acteurs et enfin de prévoir des appuis pour aider les minilaiteries à mettre en place et contrôler l'application de ces bonnes pratiques.

\section{Réflexion à mener pour que l'application des guides donne une reconnaissance de qualité à ceux qui l'appliquent}

Il est nécessaire que les acteurs de la filière, en concertation avec l'Etat, réfléchissent aux modalités de valorisation professionnelle et commerciale pour ceux qui appliquent les recommandations des guides (reconnaissance de qualité). Plusieurs pistes ont été évoquées au niveau des ateliers nationaux et de l'atelier sous-régional. Les guides pourraient servir de support à une démarche de labellisation (label de qualité reconnu par l'Etat). Ils pourraient être également utilisés comme support de la profession (obtention de cartes professionnelles liées à l'application du guide). Dans tous les cas, il a été souligné qu'il fallait également initier des campagnes d'information des consommateurs pour que les unités qui font des produits de qualité aient des retombées commerciales.

\section{Cadre pour poursuivre la concertation entre acteurs sur l'évolution de la réglementation}

La démarche expérimentée dans les deux pays a contribué à générer de nouvelles normes locales puisqu'elle a permis « de définir des pratiques d'autogestion de la qualité en concertation avec tous les acteurs et en cohérence avec les modes de consommation des différents produits » (6). Le comité de suivi qui a été mis en place lors de cette démarche pourrait devenir un cadre de concertation pour la filière lait dans les deux pays. Il a bien fonctionné parce qu'il était lié à un objectif précis et concret, ce qui n'est pas toujours le cas des cadres de concertation entre acteurs qui sont mis en place.

La concertation est un point essentiel de la démarche car elle a permis d'élaborer un outil adapté au contexte des minilaiteries et aura donc plus de chance d'être appliquée. Par la suite, ce cadre de concertation pourrait être mis à profit pour poursuivre les discussions sur un certain nombre de points précis qui ont été identifiés au cours de la démarche d'élaboration du guide : élaboration de textes au niveau national et régional (Uemoa) qui encouragent la rédaction et l'application de guides de bonnes pratiques d'hygiène, qui précisent les processus d'élaboration et les démarches officielles de validation afin que ces guides soient notamment pris en compte par les organismes de contrôle, formulation de recomman- dations pour faire évoluer la réglementation sur les produits laitiers et réviser les normes, définition de critères microbiologiques adaptés au contexte, appui à la mise en réseau des laboratoires publics et privés...

\section{Démarche qui pourrait être appliquée dans d'autres pays de l'Uemoa}

Les conditions de transformation étant assez proches dans les autres pays représentés à l'atelier sous-régional (Mali, Niger, Bénin) et dans d'autres pays d'Afrique francophone comme le Cameroun, il serait tout à fait possible d'appliquer la démarche au sein de l'Uemoa ou au sein de la Communauté économique et monétaire de l'Afrique centrale, en valorisant une partie des guides élaborés. Il convient cependant de rappeler qu'il faut tenir compte de la spécificité de chaque pays (par exemple en termes de réglementation, de zoonoses, de systèmes de transformation) et de l'importance de la démarche progressive et concertée. Le guide n'aurait en effet pas la même valeur ni le même intérêt si l'on ne suivait pas toutes les étapes (recueil des textes réglementaires, diagnostic dans les unités, rédaction du guide et validation de ces différentes étapes par un comité de suivi). La mise en place de comité de suivi est particulièrement importante dans la mesure où il contribue à définir des pratiques adaptées au contexte des petites entreprises et approuvées par tous, à favoriser une appropriation du produit final et à créer les conditions d'une concertation entre acteurs, nécessaire pour l'élaboration de politiques publiques. L'atelier sous-régional a également conclu à la nécessité d'une intégration du guide dans les politiques laitières nationales et régionales, et dans la réglementation communautaire (Uemoa).

\section{BIBLIOGRAPHIE}

1. BONFOH B., FANE A., STEINMANN P., HETZEL M., TRAORE A.N., TRAORE M., SIMBE C.F., ALFAROUKH I.A., NICOLET J., AKAKPO J.A., FARAH Z., ZINNSTAG J., 2003. Qualité microbiologique du lait et des produits laitiers vendus au Mali et leurs implications en santé publique. Etud. Rech. sahél., 8-9 : 19-27.

2. BROUTIN C., 2005. Carte du Sénégal, unités de transformation du lait local. France / Sénégal, Infoconseil / Mpea. www.infoconseil.sn

3. BROUTIN C., 2005. Liste des unités de transformation du lait au Sénégal. France / Sénégal, Infoconseil / Mpea. www.infoconseil.sn

4. DIA D., BROUTIN C., 2005. Compte-rendu de I'atelier national de restitution de la démarche d'élaboration d'un guide de bonnes pratiques d'hygiène pour la maîtrise de la qualité dans la transformation laitière. Sénégal, GRET / ministère de l'Elevage / MAE, 16 p.

5. DOLI A., NICULESCU N., 2005. Compte-rendu de I'atelier national de restitution de la démarche d'élaboration d'un guide de bonnes pratiques d'hygiène pour la maîtrise de la qualité dans la transformation laitière. Burkina Faso, GRET / ministère des Ressources animales / Chambre de commerce, d'industrie et d'artisanat / MAE, $16 \mathrm{p}$.

6. DUTEURTRE G., 2003. Normes exogènes et traditions locales : la problématique de la qualité dans les filières laitières africaines. In : Sémin. Lait sain au Sahel, Bamako, Mali, 24 fév. - 1 mars 2003.

7. FAO / OMS, n.d. Garantir la sécurité sanitaire et la qualité des aliments : Directives pour le renforcement des systèmes nationaux de contrôle alimentaire. FAO / OMS. http://www.who.int/foodsafety/ publications/fs_management/guidelines_foodcontrol/en/index.html

8. GRET, 2005. Maîtrise de la qualité dans la transformation laitière au Burkina Faso : guide de bonnes pratiques d'hygiène (comité de suivi pour I'élaboration du guide). Nogent sur Marne, France, Gret. www.gret.org

9. INFOCONSEIL, PAOA, 2005. Etat des lieux de la filière lait au Sénégal. Dakar, Sénégal, Gret / Enda graf / Paoa. www.infoconseil.sn 
10. LOMPO L., NICULESCU N., BROUTIN C., 2005. Compte-rendu de l'atelier régional de restitution de la démarche d'élaboration d'un guide de bonnes pratiques d'hygiène pour la maîtrise de la qualité dans la transformation laitière. Nogent sur Marne, France, GRET / MAE, 44 p.

11. MINISTERE DE L'AGRICULTURE ET DE LA PECHE, 1998. Les guides de bonnes pratiques d'hygiène, fiches réflexes de la direction générale de I'alimentation. Paris, France, ministère de l'Agriculture et de la Pêche.

12. MINISTERE DE L'ELEVAGE, 2005. Maîtrise de la qualité dans la transformation laitière au Sénégal : guide de bonnes pratiques d'hygiène. Dakar, Sénégal, ministère de l'Elevage. http://www.gret.org/ressource/ pdf/07686.pdf

\section{Summary}

Broutin C., François M., La Noë Niculescu N. Quality Management in Dairy Processing: Experimentation of a Common Approach to Develop Guides on Good Hygiene Practices in Senegal and Burkina Faso

After the failure of several industrial dairies, small-scale dairy processing in West Africa is developing. It helps to promote local production and meet the needs and expectations of actors. However, the success of these new small dairies depends on better quality control in these enterprises. Guides to good hygiene practices in dairy processing were elaborated in Senegal and Burkina Faso; the elaboration process was based on consultation among professionals and government services, with the collaboration of other institutional actors (laboratories, research, development, standards, consumers), all brought together to form a monitoring committee. The guides were elaborated in a series of stages validated by the monitoring committee, which provided general guidance for the study. The discussions made it possible to address the weakness of data on sanitary risks, and the necessary changes in regulations, standards and practices implemented by actors in the commodity chain. During the process of elaborating a certain number of suitable procedures and practices that the actors can apply, the viewpoints of experts and actors in the field were taken into account. If such guides are to contribute effectively to creating an environment conducive to the development of the value chain, they must be widely distributed and transposed into informative materials and training tools for all actors in the dairy value chain, support organizations, and government agencies. Reflection is also needed on how their application can provide professionals with quality recognition, such as through the creation of a collective commercial brand or labels.

Keywords: Milk - Milk product - Quality - Hygiene Senegal - Burkina Faso.
13. PROJET D'APPUI AUX FILIERES BIO-ALIMENTAIRES, 2004. Répertoire des unités de transformation de lait de Ouagadougou, Burkina Faso / Répertoire des unités de transformation de lait de Bobo-Dioulasso, Burkina Faso.

14. STANZIANI A., 2005. Histoire de la qualité alimentaire (XIXe-XXe siècle). Paris, France, Seuil, 440 p. (coll. Liber)

15. WHO, 2006. A guide to healthy food markets. Geneva, Switzerland, WHO, 42 p. http://www.who.int/foodsafety/publications/capacity/en/ index.html

Accepté le 06.11.2009

\section{Resumen}

Broutin C., François M., La Noë Niculescu N. Gestión de la calidad en la transformación lechera: experimentación de una diligencia de elaboración concertada de guías de buenas prácticas de higiene en Senegal y en Burkina Faso

La transformación de la leche a pequeña escala, se está desarrollando en Africa del Oeste. Esta permite la valorización de la producción lechera local así como responder a las necesidades y expectativas de los actores involucrados. El éxito de estas nuevas pequeñas empresas es sin embargo tributario de un mejor dominio de la calidad de estas empresas. Un intento de elaboración de guías de buenas prácticas de higiene para la transformación lechera, basada sobre la concertación entre profesionales, los servicios del Estado y con la colaboración de otros actores institucionales (laboratorios, investigación, desarrollo, normalización, consumidores), todos reunidos en el seno de un comité de seguimiento, se implementó en Senegal y en Burkina Faso. La elaboración de las guías reposó sobre una serie de etapas validadas por el comité de seguimiento, asegurando el cuadro general del estudio. Los intercambios permitieron abordar la pobreza de los datos sobre los riesgos sanitarios, la evolución necesaria de la regulación, así como las normas y prácticas implementadas por los actores de la filial. En este proceso de elaboración de un cierto número de procedimientos y de prácticas adaptadas y aplicables por los actores, se tomaron en cuenta los puntos de vista de los expertos, así como el de los actores en el campo. Para que estas guías contribuyan efectivamente a crear un medio propicio para el desarrollo de la filial, deberán ser ampliamente difundidas y transpuestas en los soportes de información y de formación para todos los actores de la filial lechera, los organismos de apoyo y los agentes del Estado. Es igualmente necesaria la reflexión sobre las modalidades que permitirán que su aplicación pueda dar a los profesionales un reconocimiento de calidad, así como la creación de una marca comercial colectiva o de licencias.

Palabras clave: Leche - Productu lácteo - Calidad - Higiene Senegal - Burkina Faso. 